Matthias Schwenkglenks

Christian Jackisch

Manuel Constenla

Joseph N. Kerger

Robert Paridaens

Leo Auerbach

André Bosly

Ruth Pettengell

Thomas D. Szucs

Robert Leonard

\section{Neutropenic event risk and impaired chemotherapy delivery in six European audits of breast cancer treatment}

Received: 17 November 2005

Accepted: 2 February 2006

Published online: 19 April 2006

(C) Springer-Verlag 2006

Impact of Neutropenia in

Chemotherapy - European (INC-EU)

Study Group.

M. Schwenkglenks · T. D. Szucs $(\bowtie)$

European Center of Pharmaceutical

Medicine, University of Basel, c/o ECPM

Executive Office,

University Hospital,

4031 Basel, Switzerland

e-mail: thomas.szucs@unibas.ch

Tel.: +41-61-2614583

Fax: +41-61-2614584

e-mail: m.schwenkglenks@unibas.ch

C. Jackisch

Department of Gynecology and

Obstetrics, Klinikum Offenbach,

Starkenburgring 66,

63069 Offenbach, Germany

e-mail: christian.jackisch@klinikum-

offenbach.de

M. Constenla

Servicio de Oncologia, Complexo

Hospitalario de Pontevedra,

36071 Pontevedra, Spain

e-mail: mconstenla@ya.com

J. N. Kerger

Service d'Oncologie, Cliniques

Universitaires UCL,

Avenue Dr. Gaston Therasse,

5530 Godinne, Belgium

e-mail: joseph.kerger@sang.ucl.ac.be

R. Paridaens

Department of Medical Oncology,

University Hospital Gasthuisberg,

Herestraat 49,

3000 Leuven, Belgium

e-mail: robert.paridaens@uz.kuleuven.ac.be

\section{Auerbach}

Universitätsklinik für Spezielle

Gynäkologie, Allgemeines

Krankenhaus (AKH) Wien,

Währinger Gürtel 18-20,

1090 Wien, Austria

e-mail: leo.auerbach@meduniwien.ac.at

A. Bosly

Service d'Hématologie, Cliniques

Universitaires UCL,

Avenue Dr. Gaston Therasse,

5530 Godinne, Belgium

e-mail: andre.bosly@sang.ucl.ac.be

R. Pettengell

St. George's Hospital Oncology Centre,

3rd Floor St. James Wing,

Blackshaw Road,

London, SW17 0QT, UK

e-mail: r.pettengell@sghms.ac.uk

\section{R. Leonard}

South West Wales Cancer Institute,

Singleton Hospital,

Sketty Lane,

Swansea, SA2 8QA, UK

e-mail: robert@swwci.vianw.co.uk

Abstract Goals of work: The aims of this study were to assess chemotherapy treatment characteristics, neutropenic event (NE) occurrence and related risk factors in breast cancer patients in Western Europe. Materials and methods: Six retrospective audits of breast cancer chemotherapy were combined into a dataset of 2,860 individuals. NEs were defined as neutropenia-related hospitalisation, dose reduction $\geq 15 \%$ or dose delay $\geq 7$ days. Summation dose intensity (SDI) was calculated to compare different types of chemotherapy regimens on a single scale. Risk factors of NE occurrence and of low relative dose intensity (RDI) $\leq 85 \%$ were identified by multiple logistic regression. Main results: Patient populations were comparable between audits. Until 1998, cyclophosphamide, methotrexate and fluorouracil regimens were most frequently used, but thereafter, anthracycline-based regimens were most common. NEs occurred in $20 \%$ of the patients and low RDI in $16 \%$. NE occurrence predicted low RDI and was associated with higher age, bigger body surface area, lower body mass index, regimen type, more chemotherapy cycles planned, normal to high SDI, concomitant radiotherapy and year of treatment. First cycle NE occurrence predicted NEs from cycle 2 onwards. A risk score using age, SDI, number of planned chemotherapy cycles and concomitant radiotherapy differentiated patients with increasing NE risk (9-37\%). An alternative score version not using concomitant radiotherapy performed moderately less well. Conclusions: NEs occurred frequently in this combined dataset and they affected treatment delivery. Identifying patients at high NE risk enables targeted prophylaxis and may avoid dose limitations.

Keywords Breast cancer - Adjuvant chemotherapy - Adverse effects · Neutropenia $\cdot$ Europe 


\section{Introduction}

Myelosuppression is a major side effect of anticancer chemotherapy. Consequences include potentially life-threatening febrile neutropenic episodes, intravenous antibiotic treatment and prolonged hospitalisation [22]. Chemotherapy dose reductions and delays are common sequelae and may affect treatment outcomes adversely $[1,18]$. In earlystage breast cancer, adjuvant chemotherapy has become an element of standard therapy and reduces the hazard rate of death by about $15 \%$ [10]. However, this benefit may be reduced or lost when relative chemotherapy dose intensity (RDI) is reduced [2-4, 23].

Trial-based reports of chemotherapy-induced neutropenia (CIN) and febrile neutropenia (FN) incidence in breast cancer patients vary widely. During the last decade, cyclophosphamide, methotrexate and fluorouracil (CMF) regimens and fluorouracil, doxorubicin or epirubicin and cyclophosphamide (FAC or FEC) regimens have been most widely used. A systematic review of randomised clinical trials with at least 50 patients per treatment arm published between 1990 and 2000 found grade III-IV CIN rates of $1-78 \%$ in CMF chemotherapy and of $3-100 \%$ in FAC or FEC chemotherapy [8]. These differences are partially explained by protocol-specific assessment rules and differences in the timing of absolute neutrophil counts (ANC) or white blood cell counts (WBC) [13]. Thus, current evidence does not always enable a specific neutropenia risk to be assigned to commonly used regimens. Detailed information on the impact of neutropenia on chemotherapy delivery in routine practice is also limited.

Prophylactic measures such as colony-stimulating factor (CSF) and anti-infectives administration can be used to avoid neutropenic event (NE) occurrence and maintain RDI. Current US and European guidelines, as well as economic constraints, recommend a limited use of such prophylaxis, and this is reflected in practice [9, 13, 21, 22]. Furthermore, new National Comprehensive Cancer Network myeloid growth factor guidelines recommend that the overall risk of neutropenia should be calculated, taking into account both patient and treatment risk factors, before deciding whether to provide growth factor support [21]. Thus, the development of clinically applicable risk models allowing prophylaxis to be targeted at high-risk patients is important. Various studies published during the last decade have addressed this, and research is currently ongoing [19]. Results are awaited from ongoing American and European prospective observational studies.

We have combined data from six retrospective European audits of breast cancer chemotherapy, enabling us to assess the incidence and extent of chemotherapy dose limitations, the incidence of NEs and the associations of both with potential risk factors with greater statistical power than the individual audits. From these analyses, we propose preliminary NE risk scores.

\section{Materials and methods}

\section{Datasets combined}

Data on NE occurrence and impaired chemotherapy delivery were obtained from six audits in which patient identification information had been removed. Details of the Chemodose 99 audit conducted in 37 centres in Belgium and Luxembourg, the Optimización del Standard de Quimioterapia Administrada en diferentes Regímenes audit conducted in 34 Spanish centres, the Audit of Primary Breast Cancer Patients conducted in 15 UK district general and teaching hospitals and an audit conducted in six German centres were reported earlier [6, 15-17]. Unpublished data came from two academic centres in Vienna, Austria (principal investigator L. Auerbach) and in Ghent, Belgium (principal investigator S. Van Belle).

In the UK audit, data were collected prospectively in $60 \%$ of the patients. All other data were collected retrospectively. Patient selection rules were designed to recruit a patient mix as seen in routine practice, but rules regarding the inclusion of stage IV patients differed between audits, and the Belgian (Ghent) study only included patients receiving adjuvant $\mathrm{CMF}$ regimens.

Variables available from all six audits comprised demographic details, diagnosis and disease stage, prognostic factors and hormone receptor status (except UK audit), planned and administered chemotherapy, toxicities, NEs and related hospitalisations, concomitant radiotherapy and CSF use. Some ANC and WBC values were missing in all audits. Comorbidity and performance status data were not available, although the latter were unlikely to be important in a mostly adjuvant setting. Limited information on long-term outcome was available from the Austrian, German and Spanish audits but not from the rest.

Coding and grouping criteria were unified. Variable definitions were compared and in discrepant or unclear cases, recalculations were performed from the cyclespecific details available. With respect to chemotherapy dose limitations, cut-off points used were $\geq 15 \%$ for reductions and $\geq 7$ days for delays. RDI was calculated as administered dose per unit time divided by planned dose per unit time. In the case of combination regimens, the RDIs for each drug were averaged. Low RDI was defined as RDI $\leq 85 \%$ [2]. NEs were defined as neutropenia-related dose delay, dose reduction or hospitalisation. The decision whether events were neutropenia-related was made by the original investigators. Due to a lack of uniform assessment rules, $\mathrm{ANC}$ or WBC data were frequently missing and thus could not be used to verify NEs for the combined dataset.

Summation dose intensity (SDI), measuring the planned dose intensity of combination regimens on a single summary scale, was calculated as proposed by Hryniuk et al. [14]. From first-line single-agent trials in metastatic breast cancer, these authors determined the unit dose intensity (UDI) required for each drug to produce a $30 \%$ 
complete plus partial response rate. For each regimen, the dose intensities of the individual agents used were expressed as fractions of their UDIs and were summed. In this study, the resulting SDI values were standardised to a recognised 'standard' of chemotherapy, namely, intravenous CMF with drug administrations on days 1 and 8 of a 28-day cycle in the following doses $\left(\mathrm{mg} \mathrm{m}^{-2}\right)$ : cyclophosphamide, 600; methotrexate, 40; 5-fluorouracil 600 [CMF 600-40-600 in days 1 and 8 for 4 weeks $(\mathrm{d} 1,8 \mathrm{ww})]$. An RDI adjusted to CMF 600-40-600 in days 1 and 8 for 4 weeks was then calculated by multiplication with the standardised SDI values (adjusted RDI).

\section{Statistical methods}

Descriptive analysis used standard statistical methods. Univariate relationships between categorical variables were assessed by chi-squared tests. Where one variable was continuous, $t$ tests and ANOVA or Mann-Whitney $U$ tests and Kruskal-Wallis tests were used, depending on the distributions observed. Where both variables were continuous, Spearman's correlation coefficients were employed because of non-normality.

Multiple logistic regression allowing for clustering by audit was used to calculate adjusted odds ratios (OR) with robust standard errors for the following outcomes: any NE occurrence, NE occurrence from cycle 2 onwards and low RDI [24].
Chemotherapy delays and dose reductions directly impacted on RDI and they were also part of the NE definition used. As a consequence of this circularity, the coefficient of the NE covariate may have been overestimated in the regression models on low RDI occurrence. Therefore, this was checked by using an alternative NE definition based on the limited ANC and WBC data available.

The independent influences on NE occurrence identified in regression analysis were used as candidate items for the development of tentative NE risk scores. Selection of score items followed the principle of achieving a maximum of predictive and discriminatory power with a minimum of complexity. Cut-off points for continuous variables were chosen empirically to optimise score performance.

Two-tailed $p=0.05$ was used to determine statistical significance. Confidence intervals (CIs) shown are at the $95 \%$ level.

\section{Results}

Patient and treatment characteristics

The Austrian, Belgian and Luxembourg, Belgian (Ghent), German, Spanish and UK audits contributed 375, 660, 82, $154,1,167$ and 422 patients (total 2,860), respectively. Of these, $79 \%$ received their chemotherapy treatments during 1995-2001.

Table 1 Patient, disease and treatment characteristics

\begin{tabular}{|c|c|c|c|c|c|}
\hline $\begin{array}{l}\text { Variable } \\
\text { Age at diagnosis (years; mean } \pm \text { SD) }\end{array}$ & $\frac{N \text { total }}{2,745^{*}}$ & $\begin{array}{l}\text { All audits } \\
51.5 \pm 11.3\end{array}$ & \multicolumn{3}{|c|}{ Inter-audit range } \\
\hline Age at diagnosis 60 years or higher $(\%)$ & $2,745^{*}$ & 24.4 & 13.6 & - & 30.7 \\
\hline Hormone receptor status ( $\%$ positive) & $2,179 * *$ & 63.7 & 56.3 & - & 70.9 \\
\hline \multicolumn{6}{|l|}{ Disease stage $* * *$} \\
\hline stage I $(\%)$ & $2,743^{*}$ & 18.1 & 14.6 & - & 21.6 \\
\hline stage III (\%) & & 15.5 & 10.4 & - & 28.9 \\
\hline stage IV (\%) & & 4.0 & 0.0 & - & 8.9 \\
\hline \multicolumn{6}{|l|}{ Chemotherapy regimen $* * * *$} \\
\hline CMF-based (\%) & $2,834 *$ & 55.7 & 47.0 & - & 72.3 \\
\hline Anthracycline-based (\%) & & 40.8 & 27.3 & - & 47.2 \\
\hline
\end{tabular}

$* N$ total $<2,860$ due to missing values spread over various datasets

$* * N$ total $<2,860$ due to inavailability of UK data and additional missing values spread over various datasets

***Inter-audit ranges of menopausal status and disease stage do not take into account the Belgian (Ghent) dataset (see text).

****Inter-audit ranges of chemotherapy regimens used do not take into account the Belgian (Ghent) audit reporting data on patients receiving CMF chemotherapy only

$C$ Cyclophosphamide, $F$ 5-fluorouracil, $M$ methotrexate 
Patient and disease characteristics were acceptably similar across audits (Table 1). However, the diagnostic spread in the Belgian (Ghent) audit was unusual with an increased proportion of stage I patients (32 vs $18 \%$ across all audits) and a reduced proportion at stage III (4 vs 16\% across all audits). Post-menopausal patients were underrepresented in this dataset, although other patient characteristics were comparable to other audits.

In total, 240 different chemotherapy regimens were planned. A comparison of these regimens was facilitated by the use of SDI [14], using CMF 600-40-600 d1,8 4w as a standard. Table 2 details the distribution of the most common regimen subtypes and their standardised SDI values, and Fig. 1 shows a histogram of standardised SDI. Mean SDI was highest in CMF chemotherapy, followed by anthracycline-based, taxane-based and other chemotherapy. Older patients generally received lower SDI regimens (correlation coefficient $-0.10, p<0.001$ ), more so in CMF $(-0.13, p<0.001)$ than in anthracyclinebased chemotherapy $(-0.07, p<0.001)$.

Concomitant radiotherapy was administered to $40 \%$ of the patients receiving CMF-based chemotherapy and to 22 , 14 and $16 \%$ of those receiving anthracycline-based, taxane-based and other regimens, respectively. CSFs were used in $12 \%$ of the patients receiving CMF-based chemotherapy and in 14, 30 and $17 \%$ of those receiving anthracycline-based, taxane-based and other regimens, respectively.
Since 1996, the use of CMF regimens and concomitant radiotherapy has decreased, while the use of anthracyclinebased regimens has increased, becoming the most frequent regimen type after 1998. The use of CSFs increased in the early 1990s and remained relatively constant after 1995 .

\section{Chemotherapy dose limitations}

Dose delays in at least one cycle occurred in $34 \%$ of the patients (inter-audit range 16-48\%), of which $8 \%$ had delays that appeared to be directly related to concomitant radiotherapy administration; whether they were preplanned chemotherapy interruptions could not be assessed. Dose reductions in at least one cycle occurred in $33 \%$ of the patients (inter-audit range 14-49\%). Forty-seven percent had either dose delays or dose reductions (inter-audit range 26-58\%). Mean $\mathrm{RDI} \pm \mathrm{SD}$ was $94 \pm 11 \%$ (inter-audit range $90 \pm 16 \%-96 \pm 8 \%$ ). Figure 2 shows details by regimen type.

RDI $\leq 85 \%$ occurred in $16 \%$ of the patients (inter-audit range $7-25 \%$ ). It occurred more frequently in CMF-based than in anthracycline-based chemotherapy (16 vs $14 \%$; $p=0.052$ ). However, anthracycline-based regimens tended to have a lower SDI than CMF-based regimens, and when RDI was adjusted to CMF 600-40-600 d1,8 4w chemotherapy, $45 \%$ of the patients fell short of the $85 \%$ threshold (inter-audit range 10-56\%).

Table 2 Frequency of use and standardised* summation dose intensity (SDI) of subtypes of planned chemotherapy regimens

\begin{tabular}{|c|c|c|c|c|c|c|}
\hline \multirow{2}{*}{$\begin{array}{l}\text { Regimen subtype } \\
\text { All regimens }\end{array}$} & \multirow{2}{*}{$\frac{N \text { total } * *}{2,832}$} & \multirow{2}{*}{$\begin{array}{l}\text { Frequency within } \\
\text { regimen type }(\%)\end{array}$} & \multirow{2}{*}{$\begin{array}{l}\text { SDI }(\text { mean } \pm \text { SD) } \\
0.92 \pm 0.19\end{array}$} & \multicolumn{3}{|c|}{ SDI inter-audit range (mean \pm SD) } \\
\hline & & & & $0.84 \pm 0.19$ & - & $1.00 \pm 0.00$ \\
\hline \multicolumn{7}{|l|}{ CMF-based regimens } \\
\hline All CMF-based & 1,578 & - & $0.94 \pm 0.17$ & $0.85 \pm 0.21$ & - & $1.02 \pm 0.10$ \\
\hline CMF, 28 day cycle & 1,012 & 64.1 & $0.99 \pm 0.11$ & $0.86 \pm 0.20$ & - & $1.07 \pm 0.00$ \\
\hline CMF, 21-day cycle & 375 & 23.8 & $0.76 \pm 0.21$ & $0.69 \pm 0.00$ & - & $1.03 \pm 0.32$ \\
\hline CMF, oral & 187 & 11.9 & $1.04 \pm 0.04$ & $1.03 \pm 0.08$ & - & $1.04 \pm 0.06$ \\
\hline CMF-based sequential & 4 & 0.3 & $0.66 \pm 0.23$ & $0.55 \pm 0.07$ & - & $1.00 \pm-$ \\
\hline \multicolumn{7}{|l|}{ Anthracycline-based regimens } \\
\hline All anthracycline-based & 1,155 & - & $0.89 \pm 0.20$ & $0.76 \pm 0.28$ & - & $0.95 \pm 0.22$ \\
\hline FAC & 194 & 16.8 & $1.05 \pm 0.12$ & $0.97 \pm 0.00$ & - & $1.05 \pm 0.07$ \\
\hline FEC & 420 & 36.4 & $0.82 \pm 0.92$ & $0.61 \pm 0.00$ & - & $0.84 \pm 0.13$ \\
\hline $\mathrm{AC}$ & 138 & 12.0 & $1.00 \pm 0.07$ & $0.90 \pm 0.20$ & - & $1.02 \pm 0.06$ \\
\hline $\mathrm{EC}$ & 154 & 13.3 & $0.77 \pm 0.29$ & $0.69 \pm 0.22$ & - & $1.00 \pm 0.46$ \\
\hline $\mathrm{A} \rightarrow \mathrm{CMF}$ & 63 & 5.5 & $0.83 \pm 0.09$ & $0.79 \pm 0.04$ & - & $1.00 \pm 0.05$ \\
\hline Anthracycline- and taxane-containing & 38 & 3.3 & $1.21 \pm 0.43$ & $0.98 \pm 0.03$ & - & $1.25 \pm 0.45$ \\
\hline Other anthracycline & 148 & 12.8 & $0.88 \pm 0.22$ & $0.79 \pm 0.10$ & - & $1.00 \pm 0.56$ \\
\hline Taxane-based & 37 & - & $0.86 \pm 0.34$ & $0.82 \pm 0.27$ & - & $1.89 \pm-$ \\
\hline Others & 62 & - & $0.70 \pm 0.08$ & $0.63 \pm 0.21$ & - & $0.70 \pm 0.07$ \\
\hline
\end{tabular}

*Standardised to intravenous CMF 600-40-600 d1,8 4w

$* * N$ total $<2,860$ due to missing values spread over various datasets

$A$ Doxorubicin, $C$ cyclophosphamide, $E$ epirubicin, $F$ 5-fluorouracil, $M$ methotrexate

$\mathrm{A} \rightarrow \mathrm{CMF}$ refers to sequential regimens where several courses of $\mathrm{A}$, then several courses of $\mathrm{CMF}$, are administered 
Fig. 1 Histogram of standardised summation dose intensity (SDI)

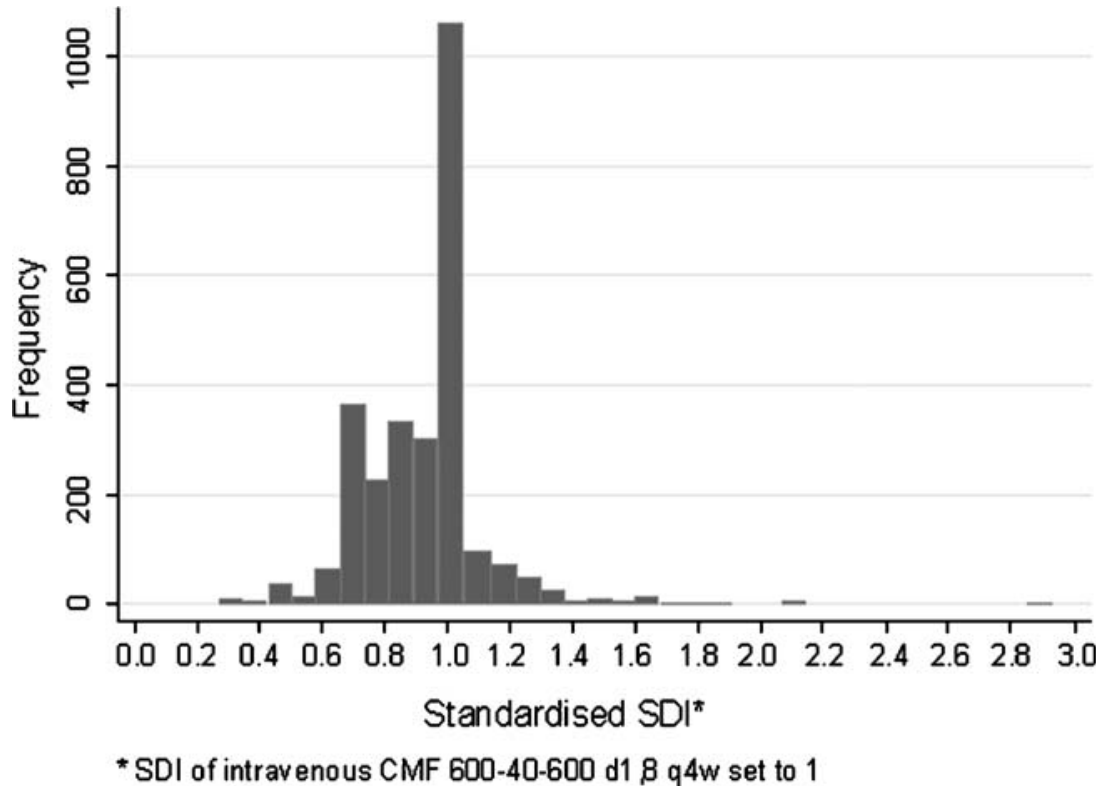

RDI was slightly lower in older patients (correlation coefficient $-0.07, p=0.001$ ). However, the proportion of patients over age 60 who received RDI $\leq 85 \%$ was not significantly higher than for patients below 60 years (17 vs $15 \%, p=0.270)$. In contrast, the proportion of patients above 60 years who received adjusted RDI $\leq 85 \%$ was higher than for younger patients (51 vs $43 \%$ below 60 years; $p<0.001)$.

\section{Neutropenic event occurrence}

NEs (neutropenia-related dose delays, dose reductions or hospitalisations) were observed in $20 \%$ of the patients (inter-audit range 15-25\%), and repeated NEs in 8\% (inter-

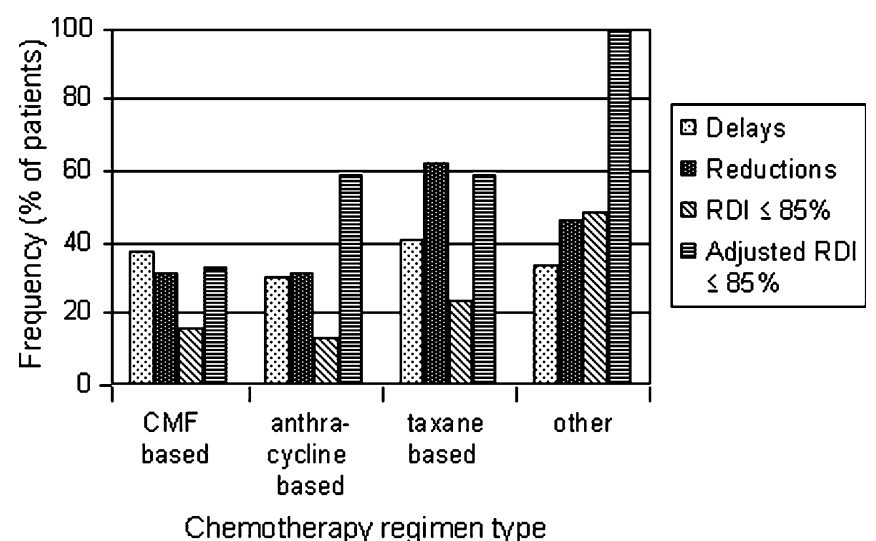

For difference between regimen types, $p=0.001$ (delays); $p<0.001$ (other parameters)

$R D I$, relative dose intensity; adjusted $R D I$, product of $R D I$ and summation dose intensity standardised to CMF 600-40-600 d1,8 4W

Fig. 2 Chemotherapy dose limitations by regimen type audit range $6-11 \%)$. Neutropenia-related dose delays were seen in 13\% (inter-audit range 6-22\%), dose reductions in $6 \%$ (inter-audit range $1-11 \%$ ) and hospitalisations in $5 \%$ (inter-audit range $4-13 \%$ ). Figure 3 shows details by regimen type.

Neutropenic event occurrence (regression results)

In logistic regression, NE occurrence was associated with higher age, higher BSA, lower BMI, regimen type, more planned chemotherapy cycles, normal to high SDI (second to fourth quartiles), concomitant radiotherapy administration and year of treatment. Concomitant radiotherapy administration interacted with BSA, number of planned chemotherapy cycles, regimen type and SDI. The change in NE risk over time was regimen-dependent. Table 3 details the model.

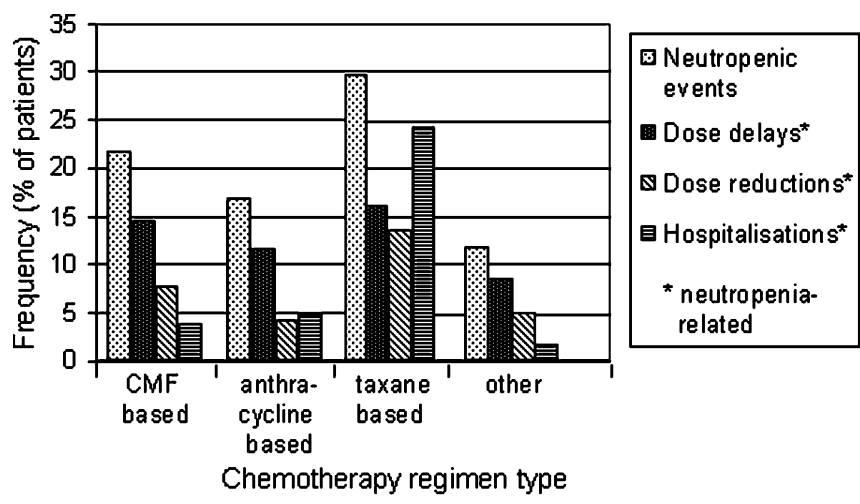

For difference between regimen types, $p=0.004$ (neutropenic events); $p=$ 0.104 (delays); $p=0.001$ (reductions); $p<0.001$ (hospitalisations)

Fig. 3 Neutropenic events by regimen type 
Table 3 Influences on any neutropenic event occurrence (logistic regression allowing for clustering by audit)

\begin{tabular}{|c|c|c|c|}
\hline \multirow{2}{*}{$\begin{array}{l}N=2,358 \\
\text { Independent variable }\end{array}$} & & \multicolumn{2}{|c|}{ Pseudo R squared of the model $=0.070$} \\
\hline & & Odds ratio $(95 \% \mathrm{CI})$ & $p^{*}$ \\
\hline Age** $^{* *}$ & & $1.02(1.01-1.03)$ & $<0.001$ \\
\hline \multirow{2}{*}{ Body surface area** } & If concomitant radiotherapy yes & $0.95(0.47-1.95)$ & 0.895 \\
\hline & If concomitant radiotherapy unknown & $13.19(2.38-73.11)$ & 0.003 \\
\hline \multirow{3}{*}{ Chemotherapy regimen $* * *$} & Anthracycline-based & $1.50(0.98-2.30)$ & 0.061 \\
\hline & Taxane-based & $1.68(1.44-1.97)$ & $<0.001$ \\
\hline & Others & $0.87(0.34-2.21)$ & 0.764 \\
\hline Normal to high SDI**** & & $1.70(1.43-2.02)$ & $<0.001$ \\
\hline Planned chemotherapy cycles** & If concomitant radiotherapy no & $1.43(1.18-1.74)$ & $<0.001$ \\
\hline \multirow{4}{*}{ Year of treatment** } & Linear, if anthracycline use & $0.85(0.75-0.95)$ & 0.007 \\
\hline & Linear, if taxane-based regimen & $1.91(1.69-2.14)$ & $<0.001$ \\
\hline & Linear, if other regimen & $1.50(1.44-1.56)$ & $<0.001$ \\
\hline & Squared & $0.99(0.98-1.00)$ & 0.075 \\
\hline \multirow[t]{7}{*}{ Concomitant radiotherapy***** } & Yes, if four-weekly CMF & $0.65(0.42-1.00)$ & 0.049 \\
\hline & Yes, if three-weekly CMF & $0.37(0.20-0.70)$ & 0.002 \\
\hline & Yes, if anthracycline-based & $0.26(0.21-0.33)$ & $<0.001$ \\
\hline & Yes, if taxane-based & - & - \\
\hline & Yes, if other regimen & - & - \\
\hline & Unknown, if four-weekly CMF & $1.01(0.01-97.00)$ & 0.995 \\
\hline & Unknown, if normal to high SDI & $1.19(0.14-10.14)$ & 0.876 \\
\hline
\end{tabular}

*Combined Wald tests, for all sets of categorical or ordinal variables and for all sets of interaction terms, $p<0.05$. Interaction effects are presented as simple effects, not as main effects plus interaction terms, for ease of interpretation

***Per one unit increase

$* * *$ Compared to four weekly CMF

$* * * *$ Second to fourth quartiles compared to first quartile

$* * * * *$ Compared to no concomitant radiotherapy administration

$* * * * * *$ Parameter estimate based on four observations, assumed to be an artefact

$C$ Cyclophosphamide, $F$ 5-fluorouracil, $M$ methotrexate, $S D I$ summation dose intensity

NE risk over time decreased for four-weekly CMF and anthracycline-based regimens, increased for taxane-based and other regimens and increased then decreased for threeweekly CMF. Three-weekly CMF had a distinctly higher NE risk compared to four-weekly CMF. Taxane-based regimens also posed a greater NE risk than four-weekly CMF, although the sample was rather small $(N=37)$. For anthracycline-based regimens, the NE risk was only slightly greater than for four-weekly CMF when time trends were taken into account.

A positive association of concomitant radiotherapy and NE occurrence was confirmed for patients receiving fourweekly CMF regimens with normal to high SDI (41\% of our sample), with an OR of 2.5 (CI 1.4-4.5; calculated from the linear predictors underlying the ORs shown in Table 3, using mean values for body surface area and number of planned chemotherapy cycles). The use of 

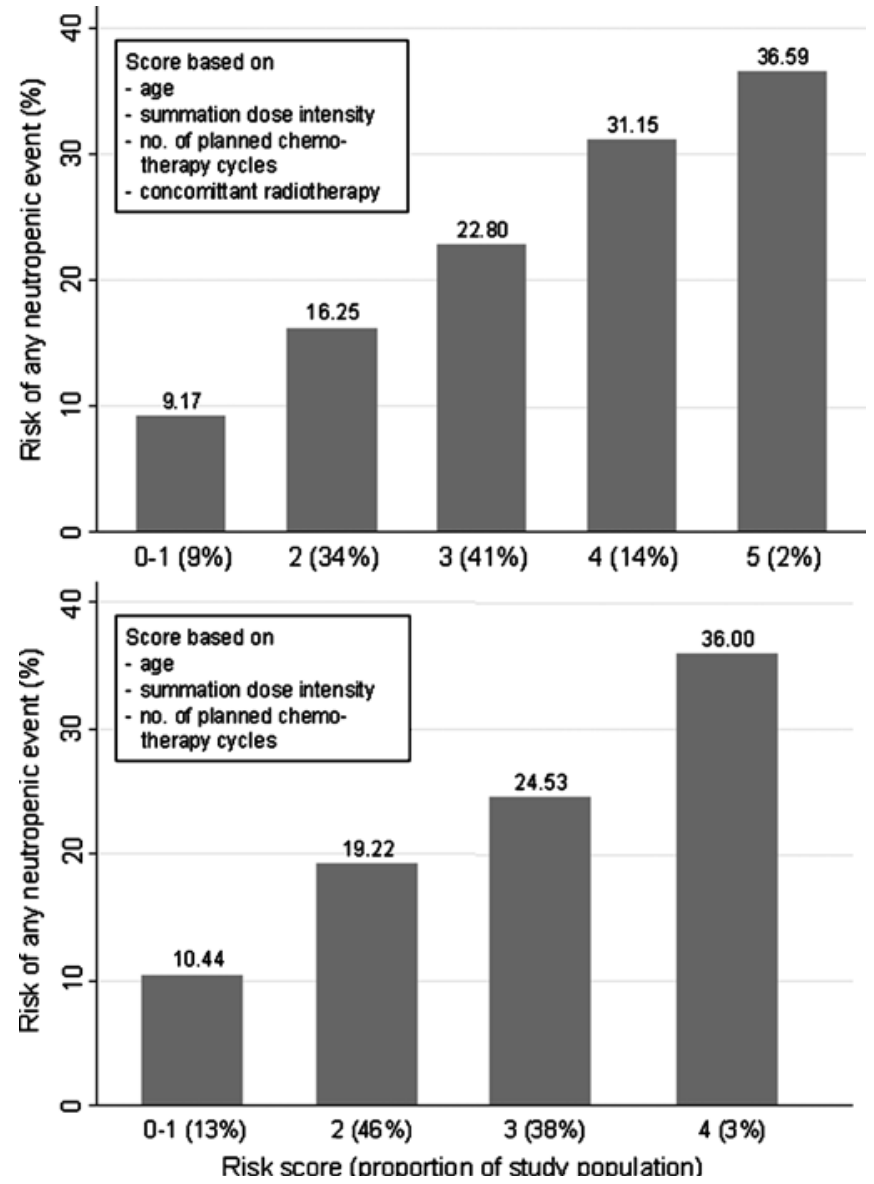

Fig. 4 Neutropenic events in any cycle by risk score

radiotherapy weakened both the association of NE risk with higher BSA and with the number of planned chemotherapy cycles.

First cycle NE occurrence was a strong predictor of NE occurrence from cycle 2 onwards, with an OR of 7.7 (CI 4.3-13.9). Otherwise, influence variables and observed interactions were as described for NE occurrence in any cycle, and coefficients were remarkably similar.

When the observations used for model estimation were restricted to patients receiving CMF- or anthracyclinebased chemotherapy, the associations and coefficients seen remained largely stable.

\section{Neutropenic event risk scores}

A drug-independent risk score based on age $(\geq 50$ vs $<50$ years), SDI (second to fourth quartiles vs lowest quartile, corresponding to a standardised SDI cut-off of 0.80 ), number of planned chemotherapy cycles $(\geq 6$ vs $<6$ ) and concomitant radiotherapy administration performed best. The score value was derived by counting the number of risk factors present and by adding 1 if there were more than six chemotherapy cycles planned. Patient groups with an increasing risk of NE occurrence in any cycle of 9-37\% were differentiated (Fig. 4, upper half). The area under the receiver operating characteristic (ROC) curve was 0.60 (CI $0.58-0.63)$. When the suggested cut-off point of more than two risk factors being present was used, sensitivity was $69 \%$ and specificity $47 \%$. Omitting concomitant radiotherapy administration as a score item reduced the area under the ROC curve to 0.57 (Fig. 4, lower half). Sensitivity was reduced to $51 \%$ and specificity increased to $61 \%$.

When first cycle NE occurrence was included as an additional score item, patient groups with an increasing risk of NE occurrence from cycle 2 onwards (8-43\%) were differentiated with sensitivity $71 \%$ and specificity $46 \%$. Omitting concomitant radiotherapy administration from this score led to a similar degradation of performance as described above for any NE occurrence.

\section{Low RDI occurrence (regression results)}

Logistic regression showed low RDI occurrence to be significantly associated with NE occurrence, higher stage of disease, regimen type, concomitant radiotherapy administration and year of treatment. When the alternative NE definition, based on the available blood cell count data, was used, the OR of low RDI for NE occurrence was reduced from 5.1 (CI 4.2-6.2) to 2.5 (CI 2.1-3.1). All other coefficients remained stable.

\section{Discussion}

This is the first multi-country study to address the incidence, risk factors and consequences of neutropenic events induced by adjuvant breast cancer chemotherapy. Six retrospective European audits of breast cancer chemotherapy, with comparable patients (similar to those seen in routine practice), were combined to generate the first transnational European database.

The most commonly followed regimens were CMF- and anthracycline-based, with administration of taxane-based and other regimens being too rare to allow reliable results. CSF use was low and followed no coordinated approach, which, from an analytical perspective, may have been advantageous because physiological relationships were not hidden by effective prophylaxis.

NEs and low RDI were confirmed to be frequent events and several independent predictors of NE occurrence were identified. SDI was demonstrated to be such a predictor, for the first time according to our knowledge. Using a cut-off point between the first and the second quartile of the SDI distribution was optimal for prediction purposes, but treating SDI as a continuous variable also produced significant results. Using SDI was crucial in separating 
the effects of regimen type from those of planned dose intensity.

A higher NE risk in three-weekly compared to fourweekly CMF was observed in the Spanish and UK audits; i.e. all audits with a substantial proportion of three-weekly CMF patients. This association has been previously reported $[12,17]$, and different centre characteristics have been proposed as a partial explanation [17]. The NE risk of anthracycline-based regimens was only moderately increased compared to four-weekly CMF, but an element of incomplete adjustment may be present in this result as we corrected for SDI but not for anthracycline dosage directly. Changes in NE risk over time and dependent upon regimen type may have been due to increasing experience and changes in medical practice.

NE risk was shown to increase linearly with age, an observation supported by earlier studies [1,26]. Correcting for SDI was important for detecting this relationship, which was partially hidden by a tendency to use lower planned dose intensity in older patients. While the association of age and NE risk appears marginal when expressed per year of age (OR 1.02; see Table 3), this corresponds to an OR of around 1.5 for an age difference of 25 years. An increased NE risk in patients with higher BSA and a protective effect of higher BMI were also demonstrated. This seems biologically plausible, particularly if it is acknowledged that BSA-based chemotherapy dosing may not be an optimal solution. The BSA effect was diluted in patients receiving concomitant radiotherapy, which might be because radiotherapy adds a BSA-independent risk component. Concomitant radiotherapy administration itself appeared to be associated with a higher NE risk in univariate analysis; in multivariate analysis, this finding was only unequivocal for four-weekly CMF regimens with normal to high SDI.

Of the variables analysed, a combination of age, SDI, number of planned chemotherapy cycles, concomitant radiotherapy administration and first cycle NE (in the case of NEs occurring from cycle 2 onwards) provided the best indicator of risk. Tentative addition of score items representing BSA, BMI or chemotherapy regimen type did not improve performance. The observed wide variety of regimen specifications, which is in part reflected in the SDI variable, and differences in practice patterns may have obscured the differences between the main types of chemotherapy regimens involved. In support of our conclusions, age, radiotherapy administration and first cycle NE have previously been used in clinical prediction models $[7,25,26]$. However, the cut-off found for age, at 50 years, may have been influenced by practice patterns and may not be universally applicable. Compared to scores derived from datasets with more baseline and first cycle haematology parameters available, our tentative scores performed only slightly less well [26]. Omitting concomitant radiotherapy, which is becoming rare, affected the performance moderately. The other parameters used, including SDI, can be expected to become important components of future risk models combining patientrelated and treatment-related factors.

An association of $\mathrm{NE}$ and low RDI was confirmed even when NEs were assessed from the limited ANC and WBC values available. Low adjusted RDI was found to occur more frequently in anthracycline-based compared to CMFbased chemotherapy due to lower SDI values in the former. In contrast, Lyman et al. reported lower SDI values in CMF- than in anthracycline-based chemotherapy in the US [20].

Our findings underline the importance of early prophylaxis. NEs are frequent and they often impact on chemotherapy delivery, with the likely effect of diminished efficacy. The importance of maintaining full chemotherapy dose intensity, in younger and older patients, has been described by several authors $[2-4,23]$. A current tendency toward dose-dense regimens may further exacerbate this problem $[5,11,27]$.

To further our current findings on the risk of NE, a prospective study measuring all potential risk factors including first cycle ANC has commenced in Europe. This will allow our findings to be validated against an external dataset, and should allow our risk score to be developed and refined to a model with increased discriminatory power. Such a risk model is becoming fundamentally important as economic constraints and current guidelines require expensive prophylactic treatments to be targeted to those at greatest risk [21].

Acknowledgements The authors wish to thank Amgen (Europe) $\mathrm{GmbH}$, Lucerne, Switzerland, for supporting this work by an unrestricted, educational grant. Thanks to Dr. Veronique Cocquyt and Prof. Simon van Belle, Department of Medical Oncology, University Hospital Ghent, Ghent, Belgium, for their permission to use the Belgian (Ghent) dataset.

\section{References}

1. Balducci L (2003) Myelosuppression and its consequences in elderly patients with cancer. Oncology (Huntingt) $17: 27-32$
2. Bonadonna G, Valagussa P, Moliterni A, Zambetti M, Brambilla C (1995) Adjuvant cyclophosphamide, methotrexate, and fluorouracil in node-positive breast cancer: the results of 20 years of follow-up. N Engl J Med 332:901-906
3. Budman DR, Berry DA, Cirrincione CT et al (1998) Dose and dose intensity as determinants of outcome in the adjuvant treatment of breast cancer. The Cancer and Leukemia Group B. J Natl Cancer Inst 90:1205-1211 
4. Chang J (2000) Chemotherapy dose reduction and delay in clinical practice: evaluating the risk to patient outcome in adjuvant chemotherapy for breast cancer. Eur J Cancer 36(Suppl 1): S11-S14

5. Citron ML, Berry DA, Cirrincione C et al (2003) Randomized trial of dosedense versus conventionally scheduled and sequential versus concurrent combination chemotherapy as postoperative adjuvant treatment of node-positive primary breast cancer: first report of Intergroup Trial C9741/Cancer and Leukemia Group B Trial 9741. J Clin Oncol 21:1431-1439

6. Constenla M, Bosly A, Jackisch C et al (2003) An audit of primary breast cancer management in Spain: the OSQAR study [abstract]. Proc Am Soc Clin Oncol 22:312

7. Crawford J, Ozer H, Stoller R et al (1991) Reduction by granulocyte colony-stimulating factor of fever and neutropenia induced by chemotherapy in patients with small-cell lung cancer. N Engl J Med 325:164-170

8. Dale DC, Crawford J, Lyman CG (2001) Chemotherapy-induced neutropenia and associated complications in randomized clinical trials: an evidencebased review [abstract]. Proc Am Soc Clin Oncol 20:1638

9. Dale DC (2002) Colony-stimulating factors for the management of neutropenia in cancer patients. Drugs 62 (Suppl 1):1-15

10. Early Breast Cancer Trialists' Collaborative Group (1998) Polychemotherapy for early breast cancer: an overview of the randomised trials. Lancet 352:930-942

11. Ellis GK, Livingston RB, Gralow JR, Green SJ, Thompson T (2002) Dosedense anthracycline-based chemotherapy for node-positive breast cancer. J Clin Oncol 20:3637-3643
12. Engelsman E, Klijn JC, Rubens RD et al (1991) "Classical" CMF versus a 3weekly intravenous CMF schedule in postmenopausal patients with advanced breast cancer. An EORTC Breast Cancer Co-operative Group Phase III Trial (10808). Eur J Cancer 27:966-970

13. ESMO Guidelines Task Force (2001) ESMO recommendations for the application of haematopoietic growth factors (hGFs). Ann Oncol 12:1219-1220

14. Hryniuk W, Frei E 3rd, Wright FA (1998) A single scale for comparing dose-intensity of all chemotherapy regimens in breast cancer: summation dose-intensity. J Clin Oncol 16: 3137-3147

15. Jackisch C, Jaber M, Burkamp U et al (2003) Maintenance of dose intensity in adjuvant chemotherapy of breast cancer in patients treated outside a clinical trial. Results of a retrospective study. Geburtshilfe Frauenheilkd 63:333-343

16. Kerger JN, Bormans V, Dauwe M (2002) Adjuvant (adj) chemotherapy (CT) delivery in patients (pts) with breast cancer (BC): results from the Chemodose Working Party BelgiumLuxembourg [abstract]. Ann Oncol 13 (Suppl 5):38

17. Leonard RC, Miles D, Thomas R, Nussey F (2003) Impact of neutropenia on delivering planned adjuvant chemotherapy: UK audit of primary breast cancer patients. Br J Cancer 89: 2062-2068

18. Link BK, Budd GT, Scott S et al (2001) Delivering adjuvant chemotherapy to women with early-stage breast carcinoma: current patterns of care. Cancer 92:1354-1367

19. Lyman GH (2003) Risk assessment in oncology clinical practice. From risk factors to risk models. Oncology (Huntingt) 17:8-13

20. Lyman GH, Dale DC, Crawford J (2003) Incidence and predictors of low dose-intensity in adjuvant breast cancer chemotherapy: a nationwide study of community practices. J Clin Oncol 21:4524-4531
21. National Comprehensive Cancer Network (NCCN) (2005) Clinical Practice Guidelines in Oncology-v.2.2005. Myeloid growth factors in cancer treatment. http://www.nccn.org/profes sionals/physician_gls/PDF/myeloid growth.pdf. Accessed May 30, 2005

22. Ozer H, Armitage JO, Bennett CL et al (2000) 2000 update of recommendations for the use of hematopoietic colony-stimulating factors: evidencebased, clinical practice guidelines. American Society of Clinical Oncology Growth Factors Expert Panel. J Clin Oncol 18:3558-3585

23. Piccart MJ, Biganzoli L, Di Leo A (2000) The impact of chemotherapy dose density and dose intensity on breast cancer outcome: what have we learned? Eur J Cancer 36(Suppl 1): S4-S10

24. Rogers WH (1993) Regression standard errors in clustered samples. Stata Tech Bull 13:19-23

25. Silber JH, Fridman M, DiPaola RS et al (1998) First-cycle blood counts and subsequent neutropenia, dose reduction, or delay in early-stage breast cancer therapy. J Clin Oncol 16: 2392-2400

26. Talcott JA, Siegel RD, Finberg R, Goldman L (1992) Risk assessment in cancer patients with fever and neutropenia: a prospective, two-center validation of a prediction rule. J Clin Oncol 10:316-322

27. Thatcher N, Girling DJ, Hopwood P et al (2000) Improving survival without reducing quality of life in small-cell lung cancer patients by increasing the dose-intensity of chemotherapy with granulocyte colony-stimulating factor support: results of a British Medical Research Council Multicenter Randomized Trial. Medical Research Council Lung Cancer Working Party. J Clin Oncol 18:395-404 